PROCEEDINGS OF THE

AMERICAN MATHEMATICAL SOCIETY

Volume 128, Number 6, Pages 1743-1749

S 0002-9939(00)05490-3

Article electronically published on February 3, 2000

\title{
ASYMPTOTIC BEHAVIOR OF SOLUTIONS OF DIFFERENCE EQUATIONS IN BANACH SPACES
}

\author{
CRISTÓBAL GONZÁLEZ AND ANTONIO JIMÉNEZ-MELADO
}

(Communicated by Hal L. Smith)

AbStRaCt. In this paper we consider the first order difference equation

$$
\Delta x_{n}=\sum_{i=0}^{\infty} a_{n}^{i} f\left(x_{n+i}\right)
$$

and give necessary and sufficient conditions so that there exist solutions which are asymptotically constant. These results generalize those given earlier by Popenda and Schmeidel. As an application we give necessary and sufficient conditions for the second order difference equation

$$
\Delta\left(q_{n} \Delta x_{n}\right)+p_{n} f\left(x_{n}\right)=0
$$

to have asymptotically constant solutions.

\section{Introduction}

The asymptotic behavior of solutions of the difference equation

$$
\Delta x_{n}=\sum_{i=0}^{r} a_{n}^{i} x_{n+i}
$$

was considered by J. Popenda and E. Schmeidel [P-S94. They gave sufficient conditions for the above equation to have asymptotically constant solutions. In S97. E. Schmeidel considered a generalization of the problem by looking at the equation

$$
\Delta x_{n}=\sum_{i=0}^{\infty} a_{n}^{i} x_{n+i} .
$$

The second order difference equation

$$
\Delta^{2} x_{n}+p_{n} f\left(x_{n}\right)=0
$$

was studied previously by A. Drozdowicz and J. Popenda [D-P87. The common point of all three papers is the use of the Schauder Fixed Point Theorem.

In this note, the results in P-S94 and S97 are generalized, and the use of the Schauder Fixed Point Theorem is replaced by the Banach Fixed Point Theorem in order to obtain a simpler proof of the existence of asymptotically constant solutions

Received by the editors July 21, 1998.

1991 Mathematics Subject Classification. Primary 39A10; Secondary 47N99.

Key words and phrases. Difference equation, asymptotic behavior.

This research was partially supported by a grant from Ministerio de Educación y Cultura (Spain) PB97-1081, and from La Junta de Andalucía. 
to the first two equations. Moreover, this study will be done under the setting of arbitrary Banach spaces since it does not present any additional complications.

As an application of our result, we study the second order difference equation

$$
\Delta\left(q_{n} \Delta x_{n}\right)+p_{n} f\left(x_{n}\right)=0,
$$

and give necessary and sufficient conditions for the existence of asymptotically constant solutions.

We will let $\mathbb{N}$ be the set of non-negative integers, $\mathbb{C}$ and $\mathbb{R}$ the fields of complex and real numbers, respectively; and $\ell_{1}(\mathbb{C})$ the space of complex-valued sequences $\left(c_{n}\right)$ such that $\left\|\left(c_{n}\right)\right\|_{1}:=\sum_{n=1}^{\infty}\left|c_{n}\right|<\infty$.

Let $\left(X,\|\cdot\|_{X}\right)$ be a complex (real) Banach space, and let $\ell_{\infty}(X)$ denote the space of all bounded sequences $\mathbf{x}=\left(x_{n}\right)$ in $X$ endowed with the norm $\|\mathbf{x}\|_{\infty}=\left\|\left(x_{n}\right)\right\|_{\infty}=$ $\sup _{n}\left\|x_{n}\right\|_{X}$. With this norm $\ell_{\infty}(X)$ is a Banach space.

We say that a sequence $\mathbf{x}=\left(x_{n}\right)$ in $X$ is asymptotically constant if there exists $x \in X$ such that $\left\|x_{n}-x\right\|_{X} \rightarrow 0$ as $n \rightarrow \infty$, in which case we say that $\mathbf{x}=\left(x_{n}\right)$ is asymptotically equal to $x \in X$.

\section{THE FIRST ORDER DIFFERENCE EQUATION}

Consider the first order difference equation

$$
\Delta x_{n}=\sum_{i=0}^{\infty} a_{n}^{i} f\left(x_{n+i}\right),
$$

where $\Delta x_{n}=x_{n+1}-x_{n}$ denotes the difference operator, the coefficients $a_{n}^{i}$ are complex numbers, and $f$ is a function from $X$ to $X$. By a solution of (5) we understand a sequence $\mathbf{x}=\left(x_{n}\right)$ in $X$ that satisfies (5).

We look for sufficient conditions on the coefficients $a_{n}^{i}$ and on the function $f$ so that (5) has solutions asymptotically constant. To each set of coefficients $\left\{a_{n}^{i}\right\}_{n, i \in \mathbb{N}}$, we associate a new set $\left\{\alpha_{n}^{j}\right\}_{n, j \in \mathbb{N}}$, as follows:

$$
\alpha_{n}^{j}=\sum_{k=0}^{j} a_{n+k}^{j-k}=a_{n+j}^{0}+a_{n+j-1}^{1}+\cdots+a_{n}^{j}, \quad n, j \in \mathbb{N} .
$$

Notice that we may regard $\alpha_{n}, n \in \mathbb{N}$, as the sequence of complex numbers $\left(\alpha_{n}^{j}\right)_{j}$.

Recall that a function $f: X \rightarrow X$ is Lipschitz if

$$
\operatorname{Lip}(f):=\sup _{\substack{x, y \in X \\ x \neq y}} \frac{\|f(x)-f(y)\|_{X}}{\|x-y\|_{X}}<\infty .
$$

Also, it is easy to see that any Lipschitz map $f: X \rightarrow X$ induces another map $\mathbf{f}: \ell_{\infty}(X) \rightarrow \ell_{\infty}(X)$, defined naturally as $\mathbf{f}(\mathbf{x})=\left(f\left(x_{n}\right)\right)$, for $\mathbf{x}=\left(x_{n}\right) \in \ell_{\infty}(X)$, and that this induced map $\mathbf{f}$ is also Lipschitz with $\operatorname{Lip}(\mathbf{f}) \leq \operatorname{Lip}(f)$.

We now proceed to state and prove the main result of this paper.

Theorem 1. Let $f: X \rightarrow X$ be a Lipschitz function and let $\left\{a_{n}^{i}\right\}_{n, i \in \mathbb{N}}$ be a set of coefficients such that for each $n \in \mathbb{N}, i d+a_{n}^{0} f$ is surjective. Assume further that the associated set $\left\{\alpha_{n}^{j}\right\}_{n, j \in \mathbb{N}}$ satisfies that for each $n \in \mathbb{N}, \alpha_{n} \in \ell_{1}(\mathbb{C})$, and $\left\|\alpha_{n}\right\|_{1} \rightarrow 0$ as $n \rightarrow \infty$. Then for each $x \in X$ there is a solution $\mathbf{x}=\left(x_{n}\right) \in \ell_{\infty}(X)$ of (5) asymptotically equal to $x$. 
Proof. Let $x \in X$ be fixed. Since $\left\|\alpha_{n}\right\|_{1} \underset{n \rightarrow \infty}{\longrightarrow} 0$, there exists $n_{0} \in \mathbb{N}$ such that

$$
\left\|\alpha_{n}\right\|_{1}<\frac{1}{2(\operatorname{Lip}(f)+1)}, \quad n \geq n_{0}
$$

Now define the operator $\mathbf{T}: \ell_{\infty}(X) \rightarrow \ell_{\infty}(X)$ as follows. For each $\mathbf{h}=\left(h_{n}\right) \in$ $\ell_{\infty}(X)$, we let $\mathbf{T} \mathbf{h}=\left((\mathbf{T} \mathbf{h})_{n}\right)$ be given by

$$
(\mathbf{T ~ h})_{n}= \begin{cases}x & \text { if } n \leq n_{0} \\ x-\sum_{j=0}^{\infty} \alpha_{n}^{j} f\left(h_{n+j}\right) & \text { if } n>n_{0}\end{cases}
$$

From the above observation, $\left(f\left(h_{n}\right)\right) \in \ell_{\infty}(X)$ whenever $\left(h_{n}\right) \in \ell_{\infty}(X)$. Thus, combining this with the fact that $\alpha_{n} \in \ell_{1}(\mathbb{C})$ for each $n \in \mathbb{N}$, it tells us that $\mathbf{T}$ is a well defined operator. We now claim that $\mathbf{T}$ is a contraction mapping. To see this, we fix $\mathbf{h}=\left(h_{n}\right), \mathbf{h}^{\prime}=\left(h_{n}^{\prime}\right) \in \ell_{\infty}(X)$, and observe that $\left\|(\mathbf{T} \mathbf{h})_{n}-\left(\mathbf{T} \mathbf{h}^{\prime}\right)_{n}\right\|_{X}=0$ for $n \leq n_{0}$, while for $n>n_{0}$,

$$
\begin{aligned}
\left\|(\mathbf{T} \mathbf{h})_{n}-\left(\mathbf{T} \mathbf{h}^{\prime}\right)_{n}\right\|_{X} & =\left\|\sum_{j=0}^{\infty} \alpha_{n}^{j} f\left(h_{n+j}\right)-\sum_{j=0}^{\infty} \alpha_{n}^{j} f\left(h_{n+j}^{\prime}\right)\right\|_{X} \\
& =\left\|\sum_{j=0}^{\infty} \alpha_{n}^{j}\left(f\left(h_{n+j}\right)-f\left(h_{n+j}^{\prime}\right)\right)\right\|_{X} \\
& \leq \operatorname{Lip}(f)\left\|\alpha_{n}\right\|_{1}\left\|\mathbf{h}-\mathbf{h}^{\prime}\right\|_{\infty} \\
& \leq \frac{\operatorname{Lip}(f)}{2(\operatorname{Lip}(f)+1)}\left\|\mathbf{h}-\mathbf{h}^{\prime}\right\|_{\infty}
\end{aligned}
$$

So

$$
\left\|\mathbf{T} \mathbf{h}-\mathbf{T} \mathbf{h}^{\prime}\right\|_{\infty} \leq \frac{1}{2}\left\|\mathbf{h}-\mathbf{h}^{\prime}\right\|_{\infty},
$$

and hence, from the Banach Fixed Point Theorem for contraction mappings, there exists a unique $\mathbf{y} \in \ell_{\infty}(X)$ such that $\mathbf{T} \mathbf{y}=\mathbf{y}$.

Observe that $\mathbf{y}=\left(y_{n}\right) \in \ell_{\infty}(X)$ is asymptotically equal to $x$, because for $n>n_{0}$,

$$
\left\|x-y_{n}\right\|_{X}=\left\|x-(\mathbf{T} \mathbf{y})_{n}\right\|_{X}=\left\|\sum_{j=0}^{\infty} \alpha_{n}^{j} f\left(y_{n+j}\right)\right\|_{X} \leq\|\mathbf{f}(\mathbf{y})\|_{\infty}\left\|\alpha_{n}\right\|_{1} \underset{n \rightarrow \infty}{\longrightarrow} 0
$$

Here, we have also used the definition of the induced map $\mathbf{f}: \ell_{\infty}(X) \rightarrow \ell_{\infty}(X)$, mentioned above.

Next, the simple relation

$$
\alpha_{n}^{j}=a_{n}^{j}+\alpha_{n+1}^{j-1}, \quad j \geq 1
$$


leads to the following relation for $n>n_{0}$ :

$$
\begin{aligned}
\Delta y_{n}=y_{n+1}-y_{n} & =(\mathbf{T} \mathbf{y})_{n+1}-(\mathbf{T} \mathbf{y})_{n} \\
& =\sum_{j=0}^{\infty} \alpha_{n}^{j} f\left(y_{n+j}\right)-\sum_{j=0}^{\infty} \alpha_{n+1}^{j} f\left(y_{n+1+j}\right) \\
& =\alpha_{n}^{0} f\left(y_{n}\right)+\sum_{j=0}^{\infty} \alpha_{n}^{j+1} f\left(y_{n+1+j}\right)-\sum_{j=0}^{\infty} \alpha_{n+1}^{j} f\left(y_{n+1+j}\right) \\
& =a_{n}^{0} f\left(y_{n}\right)+\sum_{j=0}^{\infty}\left(\alpha_{n}^{j+1}-\alpha_{n+1}^{j}\right) f\left(y_{n+1+j}\right) \\
& =a_{n}^{0} f\left(y_{n}\right)+\sum_{j=0}^{\infty} a_{n}^{j+1} f\left(y_{n+1+j}\right) \\
& =\sum_{j=0}^{\infty} a_{n}^{j} f\left(y_{n+j}\right),
\end{aligned}
$$

i.e., $\left(y_{n}\right)$ satisfies the difference equation (5) for $n>n_{0}$.

With this, define $\mathbf{x} \in \ell_{\infty}(X)$ as follows. If $n>n_{0}$, let $x_{n}=y_{n}$, and if $n \leq n_{0}$, use a recursive process to define $x_{n}$. We show how $x_{n_{0}}$ is defined. Since we want that

$$
x_{n_{0}+1}-x_{n_{0}}=\sum_{i=0}^{\infty} a_{n_{0}}^{i} f\left(x_{n_{0}+i}\right)=a_{n_{0}}^{0} f\left(x_{n_{0}}\right)+\sum_{i=1}^{\infty} a_{n_{0}}^{i} f\left(x_{n_{0}+i}\right),
$$

we use that $i d+a_{n_{0}}^{0} f$ is surjective in order to obtain $x_{n_{0}} \in X$ such that

$$
x_{n_{0}}+a_{n_{0}}^{0} f\left(x_{n_{0}}\right)=x_{n_{0}+1}-\sum_{i=1}^{\infty} a_{n_{0}}^{i} f\left(x_{n_{0}+i}\right) .
$$

Thus, $\mathbf{x}=\left(x_{n}\right)$ defined in this way is a solution of (5) in $\ell_{\infty}(X)$ asymptotically equal to $x$.

In some cases we are able to give a converse to Theorem 1, as the following shows.

Theorem 2. Let $f: X \rightarrow X$ be a continuous function and let $\left\{a_{n}^{i}\right\}$ be a set of non-negative real numbers. If $\mathbf{x}=\left(x_{n}\right) \in \ell_{\infty}(X)$ is a solution of (15) asymptotically equal to $x \in X$ and $f(x) \neq 0$, then $\alpha_{n} \in \ell_{1}(\mathbb{C}), n \in \mathbb{N}$, and $\left\|\alpha_{n}\right\|_{1} \rightarrow 0$ as $n \rightarrow \infty$.

Proof. Since $f(x) \neq 0$, by the Hahn-Banach Theorem, there exists $L \in X^{*}$ such that $L(f(x))=1$. Let $u$ be the real part of $L$.

Since $\mathbf{x}=\left(x_{n}\right)$ is asymptotically equal to $x$, the continuity of $f$ and $u$ imply that there exists $n_{0} \in \mathbb{N}$ such that $u\left(f\left(x_{n}\right)\right) \geq 1 / 2$ for all $n \geq n_{0}$.

Since $u$ is a continuous real linear functional on $X, a_{n}^{i} \geq 0$, and $\mathbf{x}=\left(x_{n}\right)$ is a solution of (5), we have for $n \geq n_{0}$

$$
u\left(x_{n+1}\right)-u\left(x_{n}\right)=\sum_{i=0}^{\infty} a_{n}^{i} u\left(f\left(x_{n+i}\right)\right) \geq \frac{1}{2} \sum_{i=0}^{\infty} a_{n}^{i},
$$


and from this we get that for all $n \geq n_{0}$,

$$
u(x)-u\left(x_{n}\right) \geq \frac{1}{2} \sum_{k=n}^{\infty} \sum_{i=0}^{\infty} a_{k}^{i}=\frac{1}{2}\left\|\alpha_{n}\right\|_{1} .
$$

This inequality implies that $\alpha_{n} \in \ell_{1}(\mathbb{C})$ for $n \in \mathbb{N}$. Furthermore, letting $n \rightarrow \infty$ the LHS goes to 0 , thus obtaining $\left\|\alpha_{n}\right\|_{1} \rightarrow 0$ as $n \rightarrow \infty$.

Remark 1. The equation (5) with $X=\mathbb{R}, f=i d$, and $a_{n}^{i}=0$ for all $i \geq r+1$ and all $n \in \mathbb{N}$ gives equation (1), which was studied by J. Popenda and E. Schmeidel in P-S94. They proved that under the assumptions $a_{n}^{0} \neq-1$ for $n \in \mathbb{N}$, and $\sum_{n=0}^{\infty}\left|a_{n}^{i}\right|<\infty$ for every $i=0, \ldots, r$, it follows that for arbitrary $C \in \mathbb{R}, C \neq 0$, there exists a solution of (1) asymptotically equal to $C$.

In this case, bearing in mind that $a_{n}^{i}=0$ for all $i \geq r+1$, we have for $j \geq r$,

$$
\begin{aligned}
\alpha_{n}^{j} & =a_{n}^{j}+a_{n+1}^{j-1}+\cdots+a_{n+j}^{0} \\
& =a_{n+j-r}^{r}+a_{n+j-r+1}^{r-1}+\cdots+a_{n+j}^{0} \\
& =a_{0}^{n+j}+a_{1}^{n+j-1}+\cdots+a_{n+j}^{0}=\alpha_{0}^{n+j},
\end{aligned}
$$

and therefore the hypotheses of Theorem 1 can be restated by saying $a_{n}^{0} \neq-1$ for $n \in \mathbb{N}, \alpha_{0} \in \ell_{1}(\mathbb{C})$, and $a_{n}^{i} \underset{n \rightarrow \infty}{\longrightarrow} 0$ for all $i=0, \ldots, r$. From here we conclude that our Theorem 1 generalizes Theorem 1 in [P-S94].

Analogously Theorem 1 also generalizes the result given by E. Schmeidel in S97. There it was proved that under the assumptions $X=\mathbb{R}, f=i d, a_{n}^{0} \neq-1$ for $n \in \mathbb{N}$, $\sup _{n \geq m}\left(\max _{i}\left|a_{n}^{i}\right|\right)>0$ for $m \in \mathbb{N}$, and $\sum_{i=0}^{\infty} \sum_{n=0}^{\infty}\left|a_{n}^{i}\right|<\infty$, then for each $K \in \mathbb{R}$ there exists a solution of (2) asymptotically equal to $K$.

Remark 2. The equation (5) with $X=\mathbb{R}, f$ continuous and $a_{n}^{i}=p_{n+i}$, where $\left(p_{j}\right)$ is a sequence of non-negative real numbers, was implicitly considered by A. Drozdowicz and J. Popenda [D-P87] in their study of the second order difference equation (3). The weaker assumption of $f$ just being continuous obliges one to use the Schauder Fixed Point Theorem, but it seems that $X=\mathbb{R}$ is a rather relevant hypothesis. It does not look obvious to us how their proof can be adapted to arbitrary Banach spaces, not even assuming $f$ compact, i.e., $f$ continuous and mapping bounded sets to relatively compact sets.

Remark 3. It was noted in [D-P87] that for $X=\mathbb{R}$ the surjectivity of $i d+\alpha f$ follows from the continuity and boundedness of $f$, by virtue of the intermediate value theorem. For $X$ infinite-dimensional the situation is different. Observe that the surjectivity of $i d+\alpha f$ is equivalent to the following condition: for any $y \in X$ the mapping $f_{y}: X \rightarrow X$ given by $f_{y}(x)=y-\alpha f(x)$ has a fixed point. This condition is satisfied for instance when $f$ is $\operatorname{Lipschitz}$ with $\alpha \operatorname{Lip}(f)<1$, or $f$ is compact with bounded range. In the former case the Banach Fixed Point Theorem applies, while in the latter we need the Schauder Fixed Point Theorem.

On the other hand, it should be noticed that for infinite-dimensional $X$ and $f: X \rightarrow X$ Lipschitz with bounded range, the mapping $i d+\alpha f$ need not be surjective. To see this, we refer to a result of P. K. Lin and Y. Sternfeld [L-St85], which ensures that if $B_{X}$ is the closed unit ball in $X$, then there exists a Lipschitz mapping $T: B_{X} \rightarrow B_{X}$ with $\inf _{x \in B_{X}}\|x-T x\|_{X}>0$. Using this and the fact that the radial projection $R$ from $X$ to $B_{X}$, given by $R(x)=x$ if $\|x\|_{X} \leq 1$ and $R(x)=x /\|x\|_{X}$ otherwise, has Lipschitz constant $\operatorname{Lip}(R) \leq 2$, we obtain that the 
function $f=\frac{1}{\alpha}(y-T \circ R), \alpha \neq 0, y \in X$, is Lipschitz with bounded range but $i d+\alpha f$ is not surjective.

\section{THE SECOND ORDER DIFFERENCE EQUATION}

We continue with the same notation as before. As an application of our result, we will consider the asymptotic behavior of the solutions to the second order difference equation

$$
\Delta\left(q_{n} \Delta x_{n}\right)+p_{n} f\left(x_{n}\right)=0,
$$

where $\left(p_{n}\right),\left(q_{n}\right)$ are sequences of complex numbers and $f$ is a function from $X$ to $X$. The equation (8) with $X=\mathbb{R}, q_{n} \equiv 1, p_{n} \geq 0$ and $f$ continuous was considered by A. Drozdowicz and J. Popenda in [D-P87. There it was shown that a necessary and sufficient (after adding the hypothesis $i d+p_{n} f$ surjective for each $n \in \mathbb{N}$ ) condition in order to obtain solutions of (8) asymptotically equal to $x$ when $f(x) \neq 0$ is

$$
\sum_{j=1}^{\infty} j p_{j}<\infty
$$

Here we give the following result, where we must assume $f$ Lipschitz in order to consider arbitrary Banach spaces.

Corollary 1. Let $f: X \rightarrow X$ be a Lipschitz function and $\left(p_{n}\right),\left(q_{n}\right)$ be two sequences of complex numbers such that

$$
\begin{gathered}
q_{n} \in \mathbb{R}^{+} \text {for all } n \in \mathbb{N} . \\
\sum_{j=0}^{\infty}\left|p_{j}\right| \sum_{k=0}^{j} \frac{1}{q_{k}}<\infty . \\
i d+\frac{p_{n}}{q_{n}} f \text { is surjective for each } n \in \mathbb{N} .
\end{gathered}
$$

Then for each $x \in X$, there is a solution $\mathbf{x}=\left(x_{n}\right) \in \ell_{\infty}(X)$ of (8) asymptotically equal to $x$.

Proof. Let $x \in X$ be fixed. Consider the difference equation

$$
\Delta x_{n}=\sum_{i=0}^{\infty} a_{n}^{i} f\left(x_{n+i}\right)
$$

where $a_{n}^{i}=\frac{p_{n+i}}{q_{n}}$. The associated set $\left\{\alpha_{n}^{j}\right\}$ of $\left\{a_{n}^{i}\right\}$ is

$$
\alpha_{n}^{j}=\sum_{k=0}^{j} a_{n+k}^{j-k}=p_{n+j} \sum_{k=0}^{j} \frac{1}{q_{n+k}} .
$$

Observe that, by (9), $\left|\alpha_{n}^{j}\right| \leq\left|\alpha_{0}^{n+j}\right|$ for all $n \in \mathbb{N}$; and by (10), $\alpha_{0} \in \ell_{1}(\mathbb{C})$. Thus $\alpha_{n} \in \ell_{1}(\mathbb{C})$ for all $n \in \mathbb{N}$, and $\left\|\alpha_{n}\right\|_{1} \underset{n \rightarrow \infty}{\longrightarrow} 0$. Therefore by Theorem $\square$ there exists $\mathbf{x}=\left(x_{n}\right) \in \ell_{\infty}(X)$, a solution of (12) asymptotically equal to $x$. From here it is easy to verify that $\mathbf{x}=\left(x_{n}\right)$ is also a solution of (8).

Following the same pattern as in the previous section, we now give a sort of converse to Corollary 1 The details are left to the reader. 
Corollary 2. Let $f: X \rightarrow X$ be a continuous function and $\left(p_{n}\right),\left(q_{n}\right)$ be two sequences of non-negative real numbers. If $\mathbf{x}=\left(x_{n}\right) \in \ell_{\infty}(X)$ is a solution of (8) asymptotically equal to $x \in X$ and $f(x) \neq 0$, then (10) holds.

\section{REFERENCES}

[D-P87] A. Drozdowicz And J. Popenda, Asymptotic Behavior of the Solutions of the Second Order Difference Equation. Proc. Amer. Math. Soc. 99-1 (1987), 135-140. MR 88d:39010

[L-St85] P. K. Lin And Y. Sternfeld, Convex Sets with the Lipschitz Fixed Point Property are compact. Proc. Amer. Math. Soc. 93 (1985), 633-639. MR 86c:47074

[P-S94] J. Popenda And E. Schmeidel, On the Asymptotic Behavior of Solutions of Linear Difference Equations. Publications Matemàtiques. 38 (1994), 3-9. MR 95j:39010

[S97] E. Schmeidel, On the Asymptotic Behaviour of Solutions of Difference Equations. Demonstratio Mathematica. XXX-1 (1997), 193-197. MR 98m:39024

Departamento de Análisis Matemático, Universidad de Málaga, Fac. Ciencias, 29071 Málaga, Spain

E-mail address: gonzalez@anamat.cie.uma.es

E-mail address: jimenez@anamat.cie.uma.es 